\title{
Short communication \\ A study to compare the effectiveness of core strengthening exercises for phase I and phase II of menstrual cycle in primary dysmenorrhea subjects
}

\author{
Zainab S. ${ }^{1}$, P. Nithyashree ${ }^{1}$, R. Jumanah ${ }^{1}$, Kamalakannan M. ${ }^{2}$, Prathap Suganthirababu ${ }^{3}$, Kumaresan A. ${ }^{4}$ \\ ${ }^{1}$ Intern, ${ }^{2}$ Assistant Professor, ${ }^{3}$ Professor, Saveetha College of Physiotherapy, Thandalam, Chennai Tamilnadu, India
}

(Received: February $2021 \quad$ Revised: April $2021 \quad$ Accepted: May 2021)

Corresponding author: Kamalakannan M. Email: Kamal1712@gmail.com

\begin{abstract}
Introduction and Aim: The common gynaecological problem among females found to be Dysmenorrhea, which is a cramping pain in the lower abdomen occurring at menstruation in the absence of any identifiable pelvic disease with a prevalence rate of $60 \%-90 \%$. The consequences of primary dysmenorrhea affect quality of life (QOL) and limitations in daily activities as well as absenteeism. There is no open literature on which phase of the menstrual cycle is better to exercise in and is more effective. The aim of this study was to compare the effectiveness of core strengthening exercises for Phase I and Phase II of menstrual cycle in primary dysmenorrhea subjects and find out which phase is best to exercise in.

Materials and Methods: A total of 150 subjects were selected using convenient sampling technique based on inclusion and exclusion criteria and randomly allotted into 2 groups (group A and group B) containing 75 subjects each. Group A (Phase I follicular phase) and group B (Phase II luteal phase) performed core strengthening exercise protocol for a duration of 3 menstrual cycles (12 weeks). Working ability, location, intensity, days of pain, dysmenorrhea (WaLIDD) score as well as EQ 5-D 5-L (Euroq15 dimension 5 level quality of life questionnaire) values were obtained before and after the intervention.
\end{abstract}

Results: An unpaired t-test of the post-test mean values of phase I and II groups show that there is a significant difference between the two groups ( $\mathrm{p}$-value $<0.0001$ )

Conclusion: From the results, it can be concluded that there is a significant difference in effectiveness of core strengthening exercises between phase I and phase II and thus exercising in Phase I is most effective.

Keywords: Menstrual cycle; primary dysmenorrhoea; physiotherapy; core muscles; exercises; core strengthening exercises.

\section{INTRODUCTION}

$\mathrm{T}$ he menstrual cycle is a regular, process that occurs in the female reproductive system that makes pregnancy possible $(1,2)$. The cycle lasts from 21-35 days with an average duration of 28 days (3). There are two phases in the menstrual cycle that last for 14 days each in a 28-day cycle. The two phases are Phase I (follicular or proliferative phase) and Phase II (luteal or secretory phase).

Primary dysmenorrhea is the pain in the lower abdomen occurring at the initial stage of menstruation (4). Affected women experience sharp intermittent spasm of pain mainly concentrated at the suprapubic region. Pain may radiate to the lower back, inner thighs and the legs. Systemic symptoms include nausea, vomiting, fatigue, diarrhea, mild fever and headache (5). Primary dysmenorrhea can be treated through pharmacological and nonpharmacological methods. Pharmacological method includes NSAIDs (non-steroidal anti-inflammatory drugs), oral contraceptives and analgesic tablets. Besides its analgesic effects NSAIDs also has many limitations such as high cost, complications, and contraindications of some drug therapies. $10 \%$ of patients being treated with NSAID's do not find the treatment effective (6). Therefore, we must look at non-pharmacological treatments, which is where physiotherapy has a role.

Several published research studies have shown that exercise reduces the intensity of pain and duration of pain in primary dysmenorrhea. Exercise releases endorphins, which are "feel good hormones" thereby, easing the symptoms of dysmenorrhea (7). As weak core muscles have been associated with, the occurrence of primary dysmenorrhea (8), corestrengthening exercises can be given. These exercises strengthen the rectus abdominus, internal and external obliques, pelvic floor muscles and erector spine, and thereby improve stability and posture and releases endorphins, which reduces the symptoms of dysmenorrhea. However, there is no open literature on comparing which menstrual phase is better to be exercising in. Thus, this study aims to investigate and compare the effects of a standard protocol of core strengthening exercises between the two phases of menstrual cycle.

\section{MATERIALS AND METHODS}

This experimental study was conducted at Saveetha College of Physiotherapy, Chennai. The WaLIDD questionnaire was distributed to students to determine 
those having primary dysmenorrhoea. Based on inclusion(students aged 18-22 years having moderate or severe primary dysmenorrhea with regular menstrual cycle) and exclusion(secondary dysmenorrhoea, irregular menstruation, history of recent surgery or trauma that would affect their ability to perform the exercise protocol) criteria, 150 subjects were selected by convenient sampling and randomly allocated into groups A and B consisting of 75 subjects each. Once signed consent was obtained, the pre-test baseline values on the quality of life questionnaire (EQ5D5L) were determined and Group A performed the core strengthening exercise protocol during the follicular phase(Phase I)and Group B performed the exercise protocol during the luteal phase(Phase II).The duration of the study was 3 menstrual cycles(12 weeks). The core strengthening exercise protocol consisted of seven exercises namely, crunches, pelvic bridges, superman exercise, bilateral single leg raises, forearm planks with reaching, dynamic side planks and cat and camel exercise. All the exercises were to be performed in one session per day and ten repetitions each. WaLIDD as well as quality of life questionnaire values were obtained after the intervention. The data collected were tabulated and statistically analysed.

\section{RESULTS}

The data obtained was analysed using unpaired t-test. When comparing the post test data of the two groups the difference was found to be significant $(p<0.0001)$. Thus, group A showed decrease in the WALIDD score and improved the quality of life more than group B.

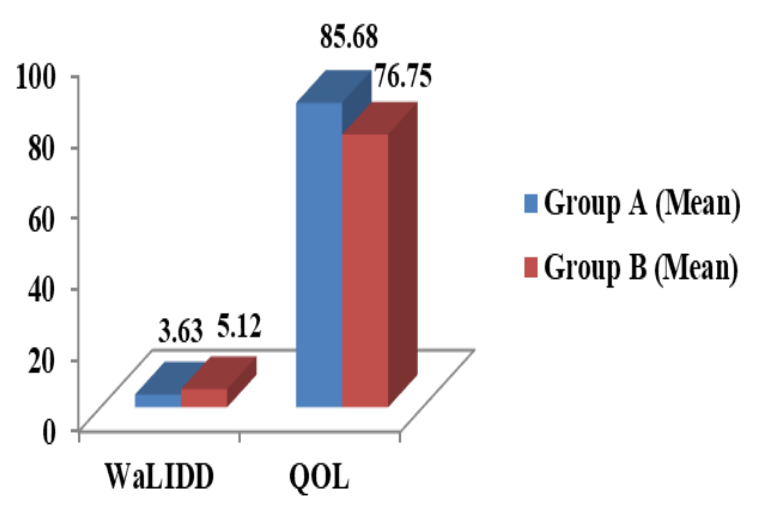

Fig. 1: Post-test data analysis of group A and group B.

WaLIDD: Working ability, location, intensity, days of pain, dysmenorrhea; QOL: Quality of life

\section{DISCUSSION}

Several studies have suggested that exercises are effective in treating primary dysmenorrhea. They reduce the pain and improve the quality of life. For instance, Matthewman et al., found that physical activity is effective in treating primary dysmenorrhea (8).
Gandhi et al., and Kaur et al., in there study they concluded that "Laura Mitchell and Physiotherapy techniques are effective for improving pain due to dysmenorrhea and there is direct correlation of core muscles weakness and pain due to primary dysmenorrhea" $(9,10)$. Therefore, core strengthening was selected to be the intervention for this study. The study duration was set as 12 weeks as several studies like Carroquino-Gracia et al., concluded that therapeutic exercise for 8-12 weeks reduce the intensity and duration of pain in primary dysmenorrhea and it also improves quality of life" (11). As there was no open literature available on which phase of the menstrual cycle is best to exercise in, this study was conducted.

It is evident from the results of this study that exercises performed during follicular phase is more effective. It can be hypothesized that due to the high levels of oestrogen and testosterone women are more energetic, and have a better mood. As women feel both physically and mentally better, this can translate into better exercise adherence and thereby improve the effects of exercise. At the end of the follicular phase is ovulation where oestrogen and testosterone are at peak levels and this augments the effects of follicular phase on energy levels and mood. In contrast to this, during the luteal phase, progesterone levels rise which in turn lead to an increase in body temperature and abdominal bloating. An imbalance of oestrogen and progesterone can affect the levels of serotonin and bring on strong premenstrual-syndrome symptoms like anxiety, depression, irritability and mood swings. Hence women may feel less inclined to perform exercises in this phase.

This study was conducted at only one university and subjects belonged to only 18-22 years of age hence the results cannot be generalized to the entire premenopausal female population. Though the results were significant, the level of adherence to the protocol was not recorded. In this study, the effects of exercise were assessed after 12 weeks and hence the long-term retention of these effects are unknown. One major limitation in this study was that the reasons for exercise producing better effects in phase I was unknown and it is recommended that a future study investigate the reasons for this.

\section{CONCLUSION}

There is a significant difference between exercising in the follicular and exercising in the luteal phases. Hence, the performance of core strengthening exercises during the follicular phase is much more effective as compared to performing them during the luteal phase of menstrual cycle.

\section{ACKNOWLEDGEMENT}

The authors are grateful to the authorities of school, parents and school teachers who rendered their full support. 


\section{CONFLICT OF INTEREST}

Authors declare no conflicts of interest.

\section{REFERENCES}

1. Human Physiology: An Integrated Approach. 6th ed. Glenview, IL: Pearson Education; 2013. ISBN 978-0-32175007-5. p. 850-890.

2. Human Physiology: From Cells to Systems. 8th ed. Belmont, California: Cengage; 2013. ISBN 978-1-11157743-8. p. 735-794.

3. Andrew S., Primary dysmenorrhea; Am Fam Physician. 1999. 1; 60(2): 489-496.

4. Khare, D., Jain, P. Effect of Different Exercise Techniques on Primary Dysmenorrhea among Higher Secondary School Girls. International Journal of Science and Research. 2016. 5(12): 2319-7064.

5. Thiyagarajan, D. K., Basit, H., Jeanmonod, R. Physiology, Menstrual Cycle. [Updated 2019 Apr 24]. In: StatPearls [Internet]. Treasure Island (FL): StatPearls Publishing; 2019.

6. Azima, S., Bakhshayesh, H. R., Abbasnia, K., Kaviani, M., Sayadi. M. Effect of isometric exercises on primary dysmenorrhea: a randomized controlled clinical trial. GMJ. 2015; 4(1): 26-32.

7. Berek, J. S., Novak, E. Berek \& Novak's Gynecology. 15th ed. Philadelphia, PA, Wolters Kluwer Health/Lippincott Williams \& Wilkins; 2012.

8. Matthewman, G., Lee, A. Physical activity for primary dysmenorrhea: a systemic review and meta-analysis of randomized controlled trials. American journal of Obstetrics and Gynecology 2018; 6(2): 603-613.

9. Kaur, M., Bains, B. S., Ramachandran, B., Rao, B. K. Role of combined exercise on primary dysmenorrhea pain among girls: a quasi-experiment. Journal of Physiotherapy Research. 2018;8(1):88-93.

10. Gandhi, N. V., Chaudhari, M., Sapan, B. S., Jhurani, B. C. Effect of lauramitchell's relaxation versus physiotherapy exercise for primary dysmenorrhea in college going students and correlation of core strength with dysmenorrhea- a randomized controlled trial. J sci tech dev. 2019; 8(10): 130140.

11. Carroquino-Garcia P., Jiménez-Rejano J. J., MedranoSanchez, E., de la Casa-Almeida, M., Diaz-Mohedo, E., Suarez-Serrano, C. Therapeutic exercise in the treatment of primary dysmenorrhea: a systematic review and metaanalysis. Phys Ther. 2019; 99: 1371. 